\title{
Maxillary restoration with complete maxillary prosthesis supported by implants with immediate loading: clinical retrospective study of $\mathbf{4 8}$ cases
}

\author{
Victor Fau ${ }^{1,{ }^{*}}$, Dany Diep ${ }^{2}$, Hervé Bénateau ${ }^{2}$, Alexis Veyssière ${ }^{2}$, Fabrice Clipet $^{1}$, \\ Patrick Limbour ${ }^{1}$ \\ ${ }^{1}$ Odontology and Oral Surgery Department, CHU, Rennes, France \\ ${ }^{2}$ Maxillofacial Surgery and Oral Surgery Department, CHU, Caen, France
}

(Received: 30 November 2016, accepted: 28 February 2017)

Keywords: immediate loading / dental implant / edentulous jaw / osseointegration

\begin{abstract}
Introduction: In recent years, the immediate loading procedure for the rehabilitation of edentulous jaws has gained popularity among patients and practitioners. The purpose of this study was to evaluate implant and prosthetic success rates for the rehabilitation of edentulous maxillae using tilted distal implants and immediate loading of prostheses, after 2 years. Material and method: Patients included in the study received a complete prosthesis of the upper arch, attached to 4, 5 or 6 implants. The provisional prosthesis was fixed the same day the implants were placed. After a period of osseointegration, the provisional prosthesis was replaced by a definitive prosthesis with a titanium framework made using computer-aided design/computer-assisted manufacturing (CAD/ CAM) technology. Judgment criteria were the implant success rate, the provisional prosthesis success rate, and the definitive prosthetic success rate. Results: Two hundred and forty-two implants were placed in 48 patients. Five implants were lost, resulting in an implant survival at 2 years of $97.9 \%$. Two provisional fixed prosthesis had to be temporarily replaced by a removable solution, resulting in a provisional prosthesis success rate of $95.8 \%$. The definitive prosthesis success rate was $100 \%$. Discussion: Use of a provisional prosthesis during the osseointegration period is essential, both for the preparation of the final prosthesis as well as for proper management of potential implant failures. Implant failures are more easily managed if at least five implants were originally placed.
\end{abstract}

\section{Introduction}

Immediate loading of supra-implanted total prosthesis, defined in 2014 by the Fifth Consensus Conference of the ITI (International Team for Implantology) as the occlusion within a maximum period of 7 days after the placement of the implants [1], has become a standard solution for the rehabilitation of the toothless arcades, both in the mandible and in the maxilla. It provides the advantage of offering quick, comfortable aesthetic and functional restoration to the patient. It also ensures strong retention between implants, limiting the amplitude of the micromovements to the bone-implant interface below the critical threshold for osseointegration [2] and decreasing the constraints transferred to the surrounding bone [3].

The primary stability of the implant is considered the most important factor for osseointegration success in immediate loading protocols $[4,5]$. The length, diameter [6], and the

\footnotetext{
* Correspondence: victor.fau@orange.fr
}

roughness of the implants $[7,8]$, as well as the associated drilling [9] are elements for achieving high insertion torque, which means good primary stability.

In the maxilla, a minimum of four implants is essential for the viability of the reconstruction. In the posterior region, where bone quality and quantity are not always optimal, surgical alternatives have been proposed: short implants [10], onlay bone graft, sinus floor elevation, or implants in specific anatomical areas such as the zygomatic bone [11], the pterygoid region [12], or the maxillary tuber [13]. Each of these techniques have their own advantages and disadvantages, but they all have limitations and require advanced surgical expertise. During the 2000s, many studies have demonstrated the interest of implant angulation in distal position [14-16]. Placed at the level of the anterior wall of the sinus, where the bone is generally dense, these implants can be longer, which provides them with better bone anchoring. Their tilt allows to increase the distance between the pillars implant mesial and distal abutments, thus decreasing the length of the prosthetic cantilever to achieve better distribution of occlusal forces [17]. 


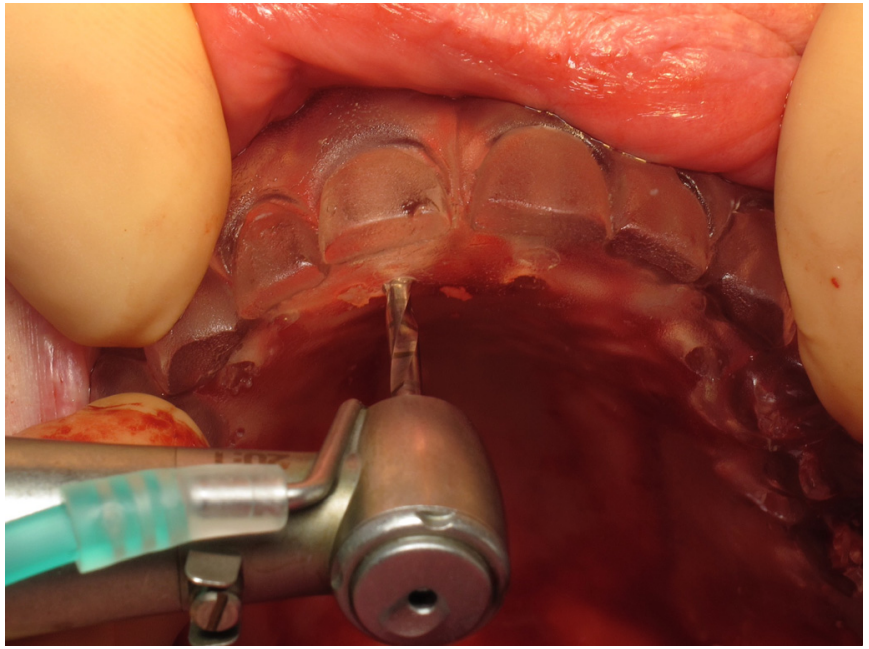

Fig. 1. Dilling through the surgical guide after elevation of the mucoperiosteal flap.

Furthermore, there is evidence that tilted implants do not exert greater direct constraint on the bone and cause no accelerated bone resorption as they are tied together by a rigid prosthesis [18].

The purpose of this retrospective study was to evaluate implant and prosthetic success rates over 2 years of rehabilitation of toothless maxillary crests, with 4,5 or 6 implants, with tilting of the two distal implants and immediate loading of the prosthesis.

\section{Material and method \\ Inclusion criteria}

The study included patients whose maxillary crest was completely toothless, who were not satisfied with their total adjoined prosthesis, and who wished to move to a fully fixed solution. Other inclusion criteria included age $>18$ years, bone volume that allowed the implementation of at least four implants of $\geq 10 \mathrm{~mm}$ length and $\geq 4 \mathrm{~mm}$ diameter, and opposing arch that allowed stable occlusal fitting. The patients included had received between June 2007 and June 2013 the placement of 4-6 maxillary implants, with immediate loading of a provisional prosthesis fixed on the day of surgery, followed by the implementation of the final prosthesis in titanium fabricated by computer-aided design and computer-aided manufacturing (CAD/CAM) after an osseointegration period of the implants.

\section{Exclusion criteria}

Exclusion criteria were the presence of inflammatory or infectious foci at the level of the implant sites, or the existence of contraindication to dental implants: risk of infective endocarditis, history of intravenous bisphosphonates use, history of irradiation of the implementation areas, active cancer or psychiatric pathology.

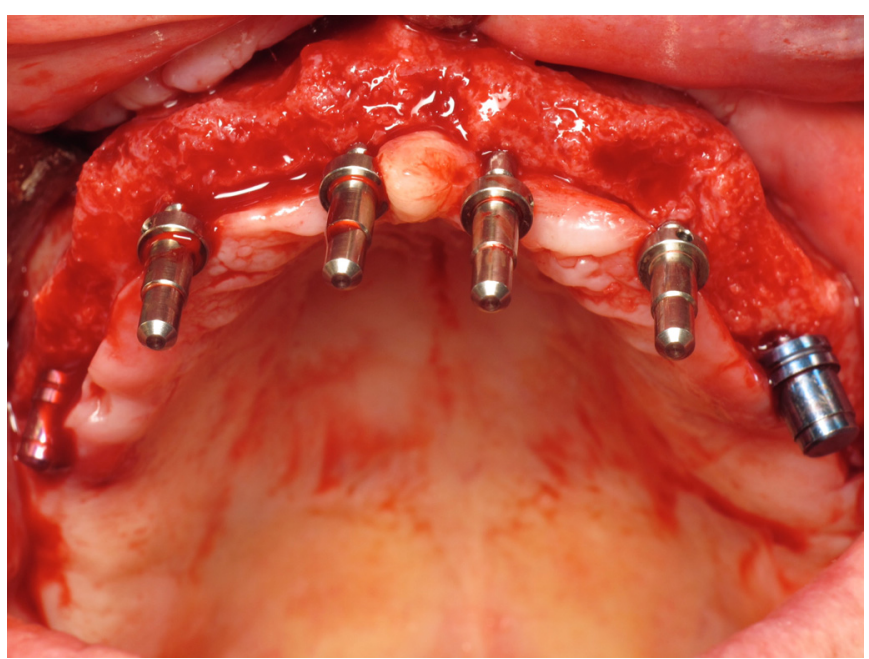

Fig. 2. Direction of drilling (notice the angulation of distal drilling).

\section{Preoperative analysis}

A preoperative evaluation was performed in all patients from a panoramic dental $X$-ray, a cone beam computed tomography of the maxilla, and mounting on the articulator of study models.

The final prosthesis played the role of steering assembly and therefore had to meet the criteria of good vertical dimension and good positioning of the teeth. If this were not the case, a new adjoined prosthesis was fabricated.

A radiological guide was obtained from a duplicate in transparent resin of the fine-tuned prosthesis, on which pits of $2 \mathrm{~mm}$ in diameter were made at the level of the ideal axes of future implants. These pits were filled with gutta-percha to be radiopaque. The maxilla cone beam was performed with the guide in place. The surgeon thus had access to a comparison between the ideal implant axes and the anatomy of the alveolar crest. The radiological guide could then be transformed into a surgical guide by removing the gutta-percha.

\section{Surgical protocol}

Premedication included amoxicillin $1 \mathrm{~g}$ twice a day starting on the morning of surgery and continued for 6 days, betamethasone $0.1 \mathrm{mg} / \mathrm{kg}$ started the morning of surgery and continued for 4 days, chlorhexidine mouthwash started the morning of surgery and continued for 10 days, and paracetamol $1 \mathrm{~g}$ in case of pain.

The interventions were carried out by two experienced oral surgeons under local anesthesia with an infiltration of articaine with adrenaline at 1/100000 (Alphacaine $\mathrm{SP}^{\oplus}$ ). After crestal incision from the molar region to the contralateral molar region, a mucoperiosteal flap was peeled, exposing the bone crest. The first drilling of each site was carried out through the surgical guide. The following drilling sequences were performed according to the standard freehand protocol, then the implants were placed in paracrestal position with insertion torque control. In case of low bone density, subjacent drilling was carried out to achieve 


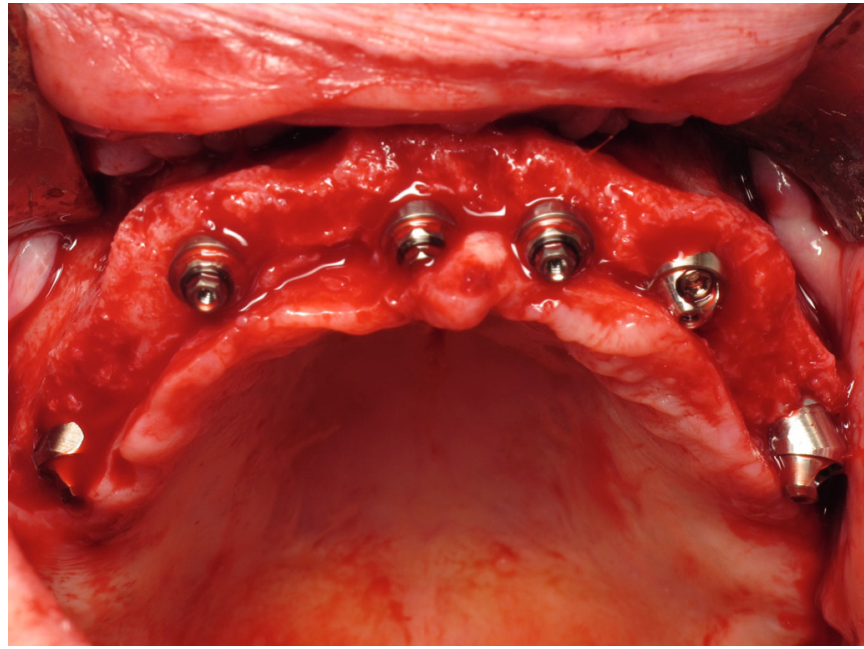

Fig. 3. Screwing of Multi-Unit Abutments ${ }^{\circledR}$ on the implants.

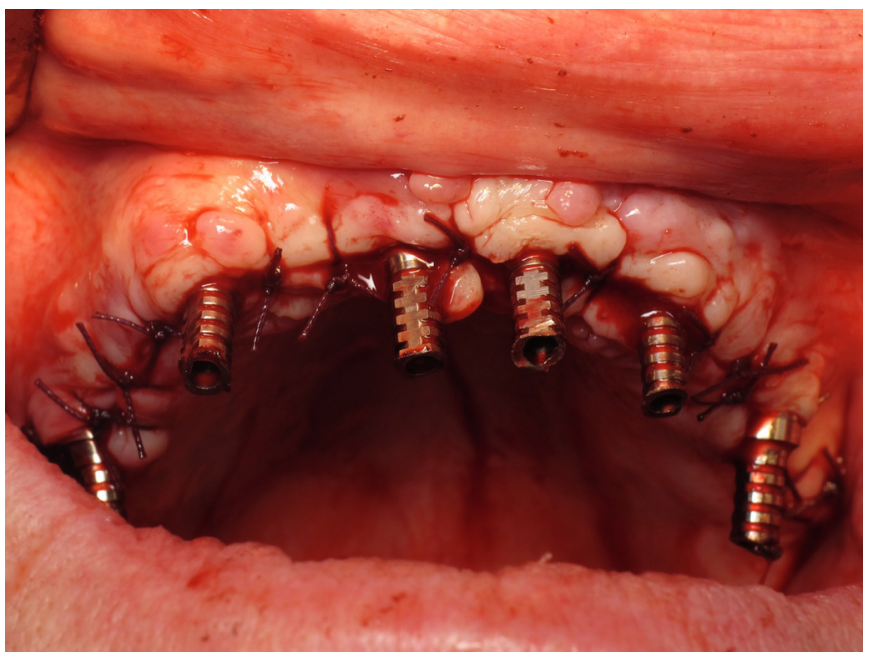

Fig. 4. Screwing of Provisional Copings ${ }^{\circledast}$ on Multi-Unit Abutments ${ }^{\circledast}$ then flap suture.

insertion torque of at least $30 \mathrm{~N} / \mathrm{cm}$. The posterior implants were tilted in the distal direction, following the anterior sinus wall and emerging more in as posterior a position as possible on the crest.

Multi-Unit Abutment ${ }^{\circledast}$ (Nobel Biocare) abutments were screwed at $15 \mathrm{~N} / \mathrm{cm}$ on the implants. These abutments were straight on the vertical implants and angulated at $17^{\circ}$ or $30^{\circ}$ on the tilted implants, to parallelize the set. Provisional cylinders (provisional Coping Multi-Unit ${ }^{\oplus}$; Nobel Biocare) were then screwed on the abutments and the mucosa was repositioned and sutured using Vicryl 3.0 slow absorption wire (Figs. 1-4).

All implants placed were NobelSpeedy ${ }^{\circledast}$ Replace RP (Nobel Biocare) of $4 \mathrm{~mm}$ diameter and $10-15 \mathrm{~mm}$ length. Those are conical implants with internal three-grooved connectivity and rough TiUnite surface achieved by anodic oxidation.

\section{Prosthetic protocol}

At this point, the adjoined final prosthesis was transformed into immediate loading prosthesis through the flaps at the level of implant emergences. Then it was positioned in the mouth and

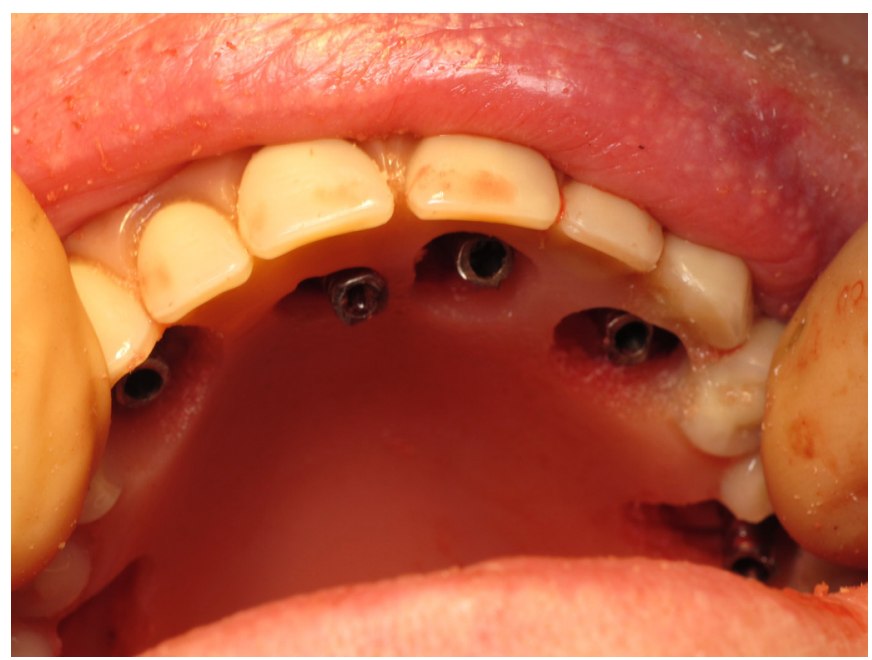

Fig. 5. Prosthesis with cappings.

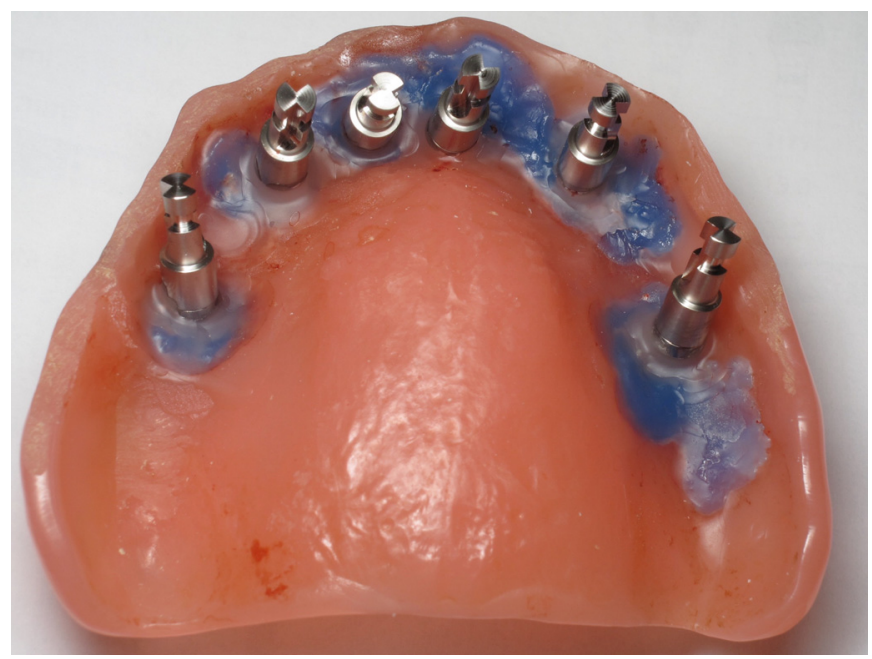

Fig. 6. Provisional copings ${ }^{\boxplus}$ unified to prosthesis.

attached to the cylinders by filling the opercula with a self-curing resin (GC Unifast Trad ${ }^{\oplus}$ ). The cylinders were then unscrewed and the prosthesis was sent to the laboratory for rework. The cylinders were trimmed at the top of the occlusal surface of the resin, the artificial palate and the vestibular band were removed, and a metal reinforcement increased the rigidity of the prosthesis.

In the end, the prosthesis was screwed in the mouth, and occlusion, aesthetics, and phonation were controlled (Figs. 5$9, \ldots, \ldots)$. A panoramic $X$-ray was obtained to check the position of the implants as well as the adaptation of prosthesis on the abutment (Fig. 10). A soft diet was recommended for 3 months.

The final prosthesis was fabricated after clinical and radiological validation of implant osseointegration, within a delay of 6-18 months after surgery. A model was made from a plaster model. The steering assembly was carried out and fitted in, inspired by the provisional prosthesis whose function and aesthetics were already validated by several months of use. The titanium superstructure was then manufactured by CAD/CAM and tested "naked" in the mouth before assembly of the cosmetic part in acrylic resin. 


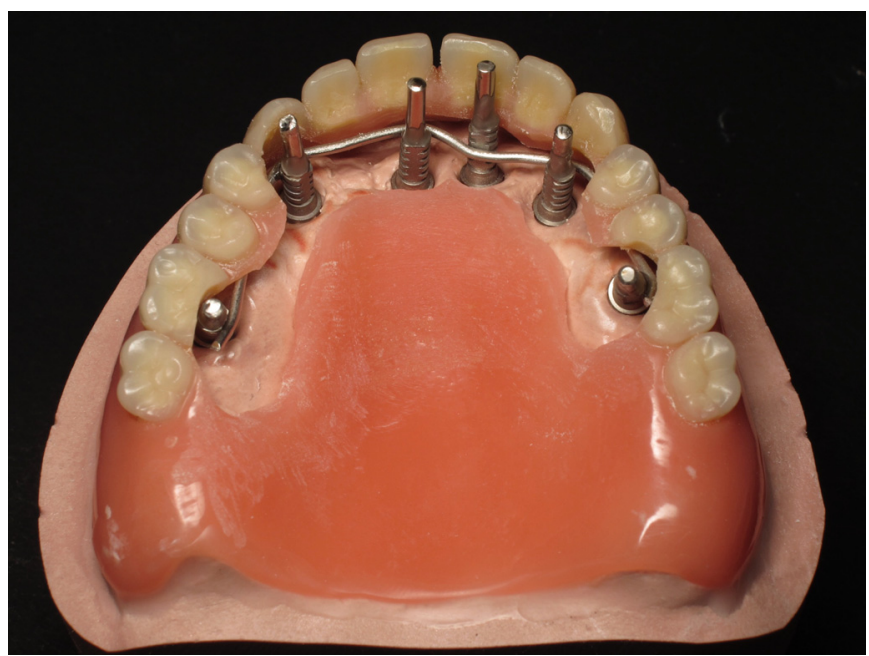

Fig. 7. Reinforcement of prosthesis with metallic bar.

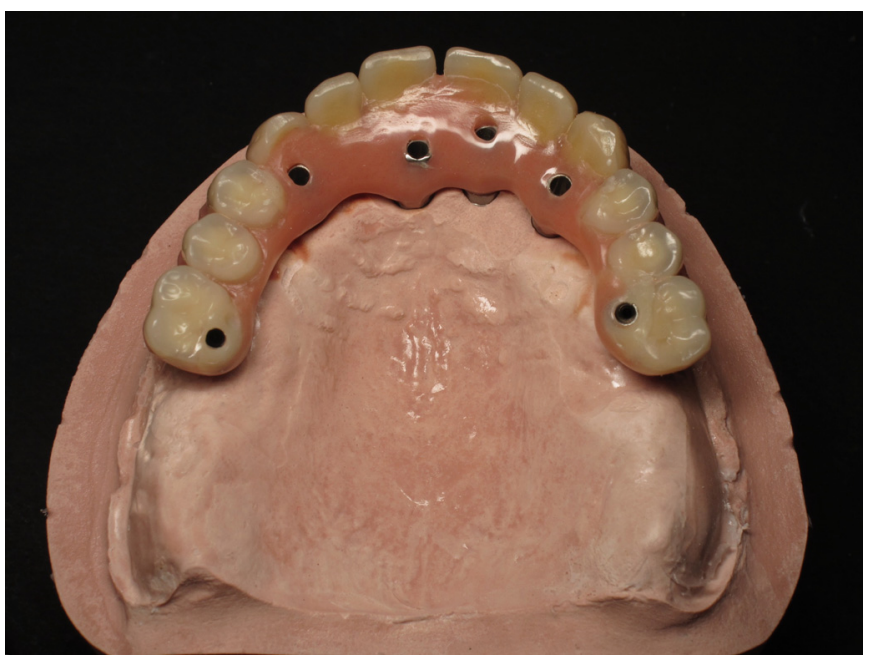

Fig. 8. Prothetic end result.

\section{Judging criteria}

Implant success: according to the criteria by Albrektson [19], an implant was classified as successful if it was motionless, free of signs of discomfort, pain, infection, neuropathy, and paresthesia's, useful to prosthetic reconstruction, without radiolucency around the implant under X-ray, and with $<2 \mathrm{~mm}$ peri-implant vertical bone loss in the first year, and with $<2 \mathrm{~mm}$ peri-implant vertical bone loss.

Provisional prosthesis success: achieved if the fixed prosthesis connected the day of the placement of the implants was retained through the implementation of the final prosthesis.

Final prosthesis success: achieved if the final prosthesis presented satisfactory aesthetics and phonatory and masticatory functions, good biological tolerance, and easy maintenance.

\section{Statistical analysis}

Statistical analyses were carried out using the software R ( $R$ Core Team) (2015). R: a language and environment for statistical computing. R Foundation for Statistical Computing,

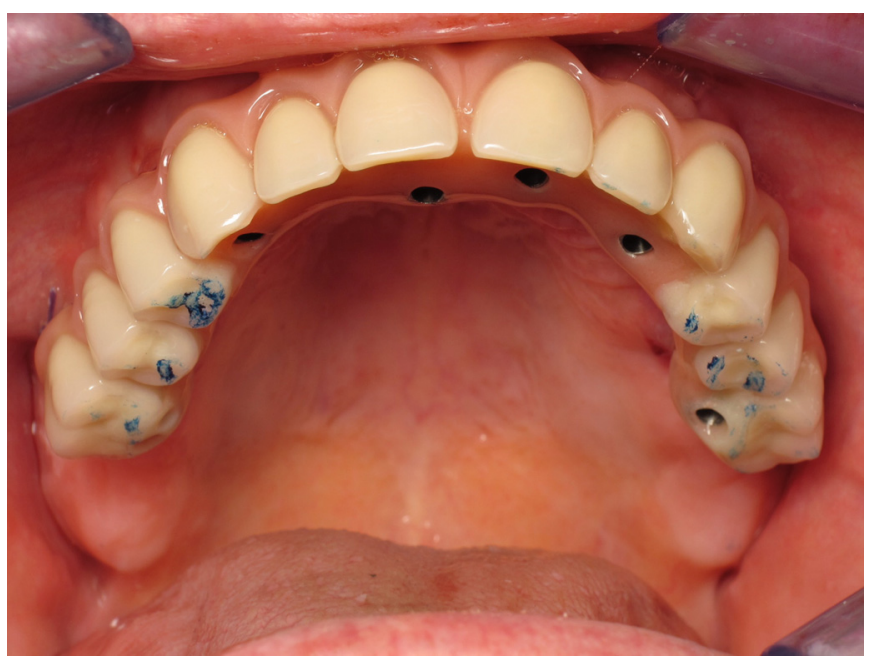

Fig. 9. Screwing of the prosthesis and occlusal check.

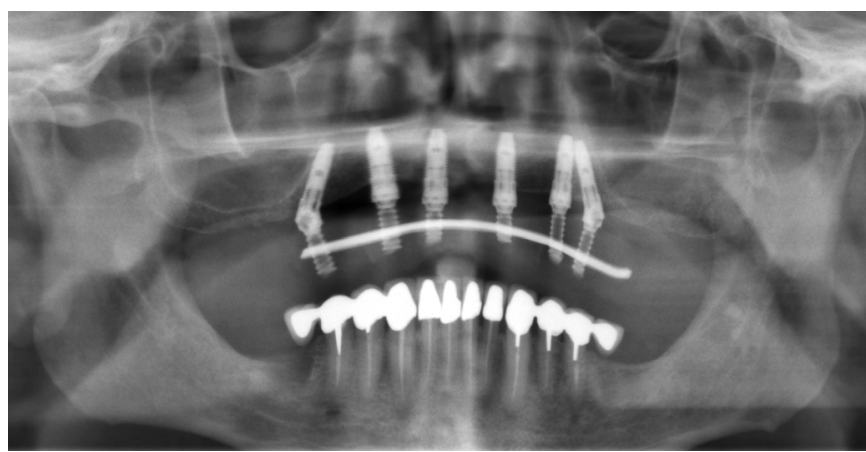

Fig. 10. Postoperative radiography.

Vienna, Austria). Two methods were used for the characterization of groups: the comparison of averages (Student $t$-test) for quantitative variables, and the $\chi^{2}$ test for qualitative variables. These analyses were made at a $5 \%$ threshold in the case of a two-tailed test.

\section{Results}

Two hundred and forty-two implants were placed in 48 patients: 26 women (54\%) and 22 men (46\%), aged $42-85$ years (mean: 64.3 years, median: 63 years). Nine patients $(19 \%)$ were smokers.

Eighteen patients $(37 \%)$ had four implants, 10 patients (21\%) had five implants, and 20 patients $(42 \%)$ had six implants. The average length of the implants was $13.16 \mathrm{~mm}$ (median: $13 \mathrm{~mm}$ ) and the average insertion torque was $44.77 \mathrm{~N} / \mathrm{cm}$ (median: $50 \mathrm{~N} / \mathrm{cm}$ ). The distribution of implant lengths, angulation and insertion torque is summarized in Table I. The opposing arch consisted of natural teeth in 37 patients $(77 \%)$, regardless of the presence of with partial adjoined prosthesis, natural teeth associated with supraimplant crowns in 6 patients $(12 \%)$, and complete fixed prosthesis on implants in 5 patients $(11 \%)$. 
Table I. Implants characteristics.

\begin{tabular}{llll}
\hline Variable & Classification & No. & $\%$ \\
\hline \multirow{2}{*}{ Angulation } & Straight & 146 & 60 \\
& Angled & 96 & 40 \\
Length $(\mathrm{mm})$ & 10 & 23 & 10.5 \\
& 11.5 & 22 & 4.5 \\
& 13 & 133 & 56 \\
& 15 & 64 & 29 \\
Torque $(\mathrm{N} / \mathrm{cm})$ & 25 & 5 & 2.1 \\
& 30 & 24 & 9.9 \\
& 35 & 20 & 8.3 \\
& 40 & 32 & 13.2 \\
& 50 & 161 & 66.5 \\
\hline
\end{tabular}

Table II. Lost implants distribution.

\begin{tabular}{|c|c|c|c|c|c|c|c|c|c|c|}
\hline $\begin{array}{l}\text { Failure } \\
\text { case } \\
\text { number }\end{array}$ & Age & Gender & Tobacco & $\begin{array}{l}\text { Number of } \\
\text { implants } \\
\text { fitted }\end{array}$ & $\begin{array}{l}\text { Length } \\
(\mathrm{mm})\end{array}$ & $\begin{array}{l}\text { Torque } \\
(\mathrm{N} / \mathrm{cm})\end{array}$ & Position & Angulation & Date of failure & Etiology of failure \\
\hline 1 & 80 years & $\mathrm{F}$ & No & 5 & 13 & 25 & Central & $0^{\circ}$ & J0 & Absence of primary fixity \\
\hline 2 & 64 years & M & Yes & 6 & 15 & 50 & Central & $0^{\circ}$ & 5 months & Osseointegration fail \\
\hline 3 & 73 years & $M$ & No & 4 & 15 & 50 & Distal & $30^{\circ}$ & 7 months & Peri-implant cratering \\
\hline 4 & 60 years & M & Yes & 4 & 15 & 30 & Distal & $30^{\circ}$ & 8 months & Peri-implant cratering \\
\hline 5 & 73 years & $M$ & No & 4 & 13 & 50 & Central & $0^{\circ}$ & 13 months & Peri-implant cratering \\
\hline
\end{tabular}

All patients had the implementation of the provisional supra-implant prosthesis on the day of surgery and the placement of the final prosthesis after a period of 6-18 months.

All patients were followed up over a period of $\geq 2$ years.

\section{Implant success}

At 2 years, five implants had to be filed (in four patients), while all the others met the predefined criteria for success, which represents an implant success rate of $97.9 \%$.

The distribution of lost implants is summarized in Table II. One implant failure occurred on the day of surgery: primary fixation was insufficient, and the implant revolved during the screwing of the supra-implant abutment. This implant was removed immediately and replaced 6 months later while the prosthesis could be supported on the remaining four implants. A failure occurred at 5 months from surgery, secondarily to fibrous integration. The three other implant failures were secondary to the early peri-implant bone cratering, and appeared between the seventh and the 13th month. All lost implants were replaced with success after a period of 4-5 months of healing.

No statistically significant relationship could be established between the implant failures and the age of patients $(p=0.18)$, gender $(p=0.15)$, tobacco consumption $(p=0.23)$, implant length $(p=0.10)$, implant angulation $(p=0.99)$, insertion torque of the implant $(p=0.28)$, the number of implants by arch $(p=0.13)$, or the type of opposing arch $(p=0.47)$.

\section{Provisional prosthesis success}

All implant failures occurred during the provisional prosthesis phase, before the production of the final prosthesis. Three implant failures (two in the same patient) referred to rehabilitation on four implants and have caused prosthetic failure, because the remaining implants were insufficient for the maintenance of the fixed prosthesis. It was thus necessary to resort in emergency to a transitional adjoined prosthetic solution. The placement of a replacement implant has secondarily allowed the return of a prosthesis fixed on four implants.

The success rate of the provisional prosthesis was $95.8 \%$.

It is worth noting that in the case of rehabilitation on five or six implants, an implant loss did not compromise the prosthetic function: the provisional prosthesis remained fixed on the remaining implants until the connection to the replacement implant.

Ten cases of partial resin fracture with detachment of a tooth were identified, caused by occlusal imbalance. Each time, the repair, complemented by adaptation of occlusion, was performed in a single session in the chair. These complications did not represent failures, but rather maintenance of the prosthesis. 
Table III. Implant success rate in complete maxillary rehabilitation with immediate loading protocol in the literature.

\begin{tabular}{llllll}
\hline Authors & $\begin{array}{l}\text { Number of implants } \\
\text { per arcade }\end{array}$ & $\begin{array}{l}\text { Number of } \\
\text { patients }\end{array}$ & $\begin{array}{l}\text { Total } \\
\text { number of } \\
\text { implants }\end{array}$ & Period of observation (months) & $\begin{array}{l}\text { Cumulative success } r \\
\text { ate (\%) }\end{array}$ \\
\hline Capelli et al. [16] & 6 & 41 & 246 & $12-40$ & 97.59 \\
Pomares [20] & 4 or 6 & 19 & 91 & 24 & 96.70 \\
Malo et al. [21] & 4 & 242 & 968 & $12-60$ & 98.00 \\
Crespi et al. [22] & 4 & 24 & 96 & 32 & 98.96 \\
Agliardi et al. [23] & 6 & 32 & 192 & 36 & 98.96 \\
\hline
\end{tabular}

Table IV. Tilted and axial implants success rate in the literature.

\begin{tabular}{|c|c|c|c|c|}
\hline Authors & Number of implants & s Angulated implants (failures) & Rights implants (failures) & Angled/right success rate (\%) \\
\hline Capelli et al. [16] & 246 & $82(2)$ & $164(3)$ & $97.56 / 98.17$ \\
\hline Malo et al. [21] & 968 & $484(12)$ & $484(7)$ & $97.52 / 98.55$ \\
\hline Crespi et al. [22] & 96 & $48(1)$ & $48(0)$ & $97.97 / 100$ \\
\hline Agliardi et al. [23] & 192 & $128(2)$ & $64(0)$ & $98.44 / 100$ \\
\hline
\end{tabular}

One patient complained of phonatory disturbances with the provisional prosthesis. The problem was solved during the transition to the final prosthesis. Finally, a patient was not satisfied with the aesthetic rendering of the provisional prosthesis. Again, the problem was solved with the final prosthesis.

\section{Final prosthesis success}

No final prosthesis had to be filed and all had satisfactory aesthetics and masticatory and phonatory functions, as well as easy maintenance. Final prosthetic success rate at 2 years was $100 \%$.

\section{Discussion}

The high implant success rate $(97.9 \%)$ achieved in our study is in agreement with the previously published data $[16,20-23]$ (Table III). The study thus confirms the excellent results achieved with the protocols of immediate loading of prostheses supported by a combination of 4-6 axial and angulated implants. According to the systematic literature review by Papaspyridakos et al., the implant success obtained with protocols of immediate loading of total supra-implant prosthesis does not present a statistically significant difference from early or conventional loading protocols [5].

Among the parameters assessed in our study, none revealed any statistically significant association with the risk of implant failure. However, the literature suggests that smoker patients must be informed of a higher risk of implant failure. According to a recent meta-analysis [24], smoking consumption statistically affects implant success rate $(p<0.00001)$ with an odds ratio of 2.23 (CI 95\% [1.96; 2.53]) compared to nonsmoker patients. Tobacco increases the incidence of postoperative infections as well as peri-implant bone loss. In our study, the implant failure rate was higher in smokers ( $4.3 \%$ against $1.5 \%$ in nonsmokers), but the difference was not significant $(p=0.23)$ because of the lack of statistical power.

It is worth noting the lack of difference regarding the $n$ success rates between angulated and straight implants in our study (success rate of $97.9 \%$ for both angulated and straight implants; $p=0.99)$. This result, according to literature data [16,21-23] (Table IV) validates the viability of angulated implants, even those placed in a region where bone density is usually low. Angulated implants offer an additional advantage to decrease distal prosthetic extensions while maintaining satisfactory arch length and good distribution of the mechanical stresses transmitted to the prosthetic frame and the peri-implant bone [17], all the while freeing elevations of low sinus depth, thus decreasing the time and cost of treatment. It has been reported that shorter prosthetic extension lengths $(<15 \mathrm{~mm})$ were correlated to an increase in prosthetic survival [25].

An insertion torque of at least $30 \mathrm{~N} / \mathrm{cm}$, which equals sufficient primary stability, is an essential precondition to immediate loading according to the 2014 ITI Consensus Conference [1]. The use of long implants (average length $13.16 \mathrm{~mm}$ ) and one subjacent drilling when bone density was low have enabled to achieve this value in $97.9 \%$ implants (average $44.77 \mathrm{~N} / \mathrm{cm}$ ). However, despite these precautions, five implants $(2.1 \%)$, in five different patients, featured an insertion torque of $25 \mathrm{~N} / \mathrm{cm}$. Of these, four were connected to the prosthesis the day of surgery while the fifth was removed in the preoperative period due almost null fixity. These four implants were subsequently osseointegrated. Thus, if in individual rehabilitation, an insertion torque of $<30 \mathrm{~N} / \mathrm{cm}$ 
may be a contraindication to immediate implant loading, presumably that is not the case in total rehabilitation, insofar as the implant is connected to other implants with higher primary fixity through the immediate prosthesis, which plays the role of rigid restraints and allows to decrease implant micromovements [2,3].

It is interesting to note that in our study, the majority of implant failures occurred in the first months following the intervention. A literature review by Patzelt et al. [26] on rehabilitation by prostheses on stilts regarding 4804 implants shows that $74 \%$ implant failures have occurred during the first 12 months. This is a percentage close to the $80 \%$ failure rate before 12 months found in our study. Similarly, Malo et al. [21] shows $89 \%$ implant failures in 13 months following surgery. These figures show that the osseointegration period in the first postoperative months is the most conducive period to the implant failure. The use of a provisional prosthesis during this period will allow for simple adaptation in case an implant should be replaced. Provided that at least four residual implants persist, the maintenance of the fixed prosthesis can be ensured through its connection to the replacement implant, placed 4-6 months after the removal of the lost implant. This situation was found twice in our study. The observation is not valid in the case where only four implants are initially in place, as the three residual implants are insufficient to maintain the fixed prosthesis in sustainable conditions. In this case, it would be fitting to use an adjoined prosthesis during the time to place the replacement implant. This situation was found twice in our study. Particularly painful for the patient, it is a strong argument in favor of placing at least five implants. Although the number of implants in maxillary reconstruction is rarely discussed in the literature, this argument is found in the literature revue by Lambert et al. [27].

In any case, if an implant failure occurs while the final prosthesis is in place, it must be removed and the titanium frame must be fully rebuilt, causing significant delays and financial cost.

As in the literature [20-22], most prosthetic complications identified in our study are represented by the resin fractures on the provisional prosthesis, which occurred in 10 patients (20.8\% cases). They bear witness to the importance of good occlusal control insofar as the absence of periodontal around the implants strongly decreases proprioceptive abilities, and therefore detection of occlusal overload.

The occlusal scheme applied was that of bilaterally balanced occlusion with simultaneous anterior and posterior contacts in centered and excursion relationship. Although no study proves the superiority of this technique, a majority of authors agree that this scheme allows to minimize the most the traction forces applied to implants and to spread the compressive forces over the whole of the arch [28]. To steer the most the masticatory forces in the axis of the implants, lateral stress and intercuspation interference must be decreased by limiting the heights and intercuspation slopes as well as the depth of the fossae [29].
The success rate of the final prosthesis achieved in the study remains $100 \%$, as in the majority of published studies $[16,20,22]$.

\section{Conclusion}

Prosthetic implant rehabilitation of toothless maxillary arches by a combination of axial and tilted implants with immediate loading of the prosthesis on stilts shows excellent results after 2 years of service when done by an experienced surgical team. The use of a provisional prosthesis is an essential path to ensure the success of the treatment.

Conflicts of interest: The authors declare that they have no conflicts of interest in relation to this article.

\section{References}

1. Gallucci G0, Benic GI, Eckert SE, Papaspyridakos P, Schimmel M, Schrott A, et al. Consensus statements and clinical recommendations for implant loading protocols. Int $\mathrm{J}$ Oral Maxillofac Implants 2014;29(Suppl):287-290.

2. Tarnow DP, Emtiaz S, Classi A. Immediate loading of threaded implants at stage 1 surgery in edentulous arches: ten consecutive case reports with 1- to 5-year data. Int J Oral Maxillofac Implant 1997;12:319-324.

3. Bergkvist G, Simonsson K, Rydberg K, Johansson F, Dérand T. A finite element analysis of stress distribution in bone tissue surrounding uncoupled or splinted dental implants. Clin Implant Dent Relat Res 2008;10:40-46.

4. Javed F, Romanos GE. The role of primary stability for successful immediate loading of dental implants. A literature review. J Dent 2010;38:612-620.

5. Papaspyridakos P, Chen C-J, Chuang S-K, Weber H-P. Implant loading protocols for edentulous patients with fixed prostheses: a systematic review and meta-analysis. Int J Oral Maxillofac Implants 2014;29(Suppl):256-270.

6. Ding X, Liao S-H, Zhu X-H, Zhang X-H, Zhang L. Effect of diameter and length on stress distribution of the alveolar crest around immediate loading implants. Clin Implant Dent Relat Res 2009;11:279-287.

7. Gotfredsen K, Wennerberg A, Johansson C, Skovgaard LT, Hjørting-Hansen E. Anchorage of Ti02-blasted, HA-coated, and machined implants: an experimental study with rabbits. J Biomed Mater Res 1995;29:1223-1231.

8. Cochran DL, Schenk RK, Lussi A, Higginbottom FL, Buser D. Bone response to unloaded and loaded titanium implants with a sandblasted and acid-etched surface: a histometric study in the canine mandible. J Biomed Mater Res 1998;40:1-11.

9. Degidi M, Daprile G, Piattelli A. Influence of underpreparation on primary stability of implants inserted in poor quality bone sites: an in vitro study. J Oral Maxillofac Surg Off J Am Assoc Oral Maxillofac Surg 2015;73:1084-1088.

10. Renouard F, Nisand D. Short implants in the severely resorbed maxilla: a 2-year retrospective clinical study. Clin Implant Dent Relat Res 2005;7(Suppl 1):104-110.

11. Chrcanovic BR, Abreu MHNG. Survival and complications of zygomatic implants: a systematic review. Oral Maxillofac Surg 2013;17:81-93. 
12. Balshi TJ, Wolfinger GJ, Balshi SF. Analysis of 356 pterygomaxillary implants in edentulous arches for fixed prosthesis anchorage. Int $\mathrm{J}$ Oral Maxillofac Implants 1999;14: 398-406.

13. Lopes LF dT P, da Silva VF, Santiago JF, Panzarini SR, Pellizzer EP. Placement of dental implants in the maxillary tuberosity: a systematic review. Int J Oral Maxillofac Surg 2015;44: 229-238.

14. Aparicio C, Perales P, Rangert B. Tilted implants as an alternative to maxillary sinus grafting: a clinical, radiologic, and periotest study. Clin Implant Dent Relat Res 2001;3:39-49.

15. Krekmanov L, Kahn M, Rangert B, Lindström H. Tilting of posterior mandibular and maxillary implants for improved prosthesis support. Int J Oral Maxillofac Implants 2000;15:405-414.

16. Capelli M, Zuffetti F, Del Fabbro M, Testori T. Immediate rehabilitation of the completely edentulous jaw with fixed prostheses supported by either upright or tilted implants: a multicenter clinical study. Int J Oral Maxillofac Implants 2007;22:639-644.

17. Bevilacqua M, Tealdo T, Menini M, Pera F, Mossolov A, Drago C, et al. The influence of cantilever length and implant inclination on stress distribution in maxillary implant-supported fixed dentures. J Prosthet Dent 2011;105:5-13.

18. Del Fabbro M, Ceresoli V. The fate of marginal bone around axial vs. tilted implants: a systematic review. Eur J Oral Implantol 2014;7(Suppl 2):171-189.

19. Albrektsson T, Jansson T, Lekholm U. Osseointegrated dental implants. Dent Clin North Am 1986;30:151-174.

20. Pomares C.A retrospective clinical study of edentulous patients rehabilitated according to the "all on four" or the "all on six" immediate function concept. Eur J Oral Implantol 2009;2:55-60.
21. Maló P, de Araújo Nobre M, Lopes A, Francischone C, Rigolizzo M. "All-on-4" immediate-function concept for completely edentulous maxillae: a clinical report on the medium (3 years) and longterm (5 years) outcomes. Clin Implant Dent Relat Res 2012;14 (Suppl 1):e139-e150.

22. Crespi R, Vinci R, Capparé P, Romanos GE, Gherlone E. A clinical study of edentulous patients rehabilitated according to the "all on four" immediate function protocol. Int J Oral Maxillofac Implants 2012;27:428-434.

23. Agliardi EL, Pozzi A, Stappert CFJ, Benzi R, Romeo D, Gherlone E. Immediate fixed rehabilitation of the edentulous maxilla: a prospective clinical and radiological study after 3 years of loading. Clin Implant Dent Relat Res 2014;16:292-302.

24. Chrcanovic BR, Albrektsson T, Wennerberg A. Smoking and dental implants: a systematic review and meta-analysis. J Dent 2015; 43:487-498.

25. Shackleton JL, Carr L, Slabbert JC, Becker PJ. Survival of fixed implant-supported prostheses related to cantilever lengths. J Prosthet Dent 1994;71:23-26.

26. Patzelt SBM, Bahat 0, Reynolds MA, Strub JR. The all-on-four treatment concept: a systematic review. Clin Implant Dent Relat Res 2014;16:836-855.

27. Lambert FE, Weber H-P, Susarla SM, Belser UC, Gallucci GO. Descriptive analysis of implant and prosthodontic survival rates with fixed implant-supported rehabilitations in the edentulous maxilla. J Periodontol 2009;80:1220-1230.

28. Bocklage R. Biomechanical aspects of monoblock implant bridges for the edentulous maxilla and mandible: concepts of occlusion and articulation. Implant Dent 2004;13:49-53.

29. Nikolopoulou F, Ktena-Agapitou P. Rationale for choices of occlusal schemes for complete dentures supported by implants. J Oral Implantol 2006;32:200-203. 